\title{
A TRACKING ROBUST LEARNING CONTROL FOR MICRO SCALE ACTUATOR SYSTEMS
}

\author{
Ali Al-Ghanimi ${ }^{1}$, Abdal-Razak Shehab ${ }^{1}$, Adnan Alamili ${ }^{1}$ \\ ${ }^{1}$ University of Kufa, Faculty of Engineering, Electrical Department \\ Emails:alih.alghanimi@uokufa.edu.iq, abdulrazzaq.aljuburi@uokufa.edu.iq,adnan.alaamili@uokufa.edu.iq
}

\begin{abstract}
In this paper, a robust sliding mode-based learning control (RSMLC) scheme is developed for a micro-scale actuator (MSA) system. By exploiting the instantaneous information of the system closed-loop stability, a sliding mode controller based on the recursive learning technique is designed. The proposed control approach can govern the sliding variable and tracking error between the desired references and actual displacement of the MSA to asymptotically converge to zero. Unlike conventional sliding mode control (CSMC), no prior knowledge of MSA system uncertainties and parameters variation is required in the RSMLC design. Besides, there is no explicit switching element in the RSMLC structure, it can be considered as a chattering free control method. Meanwhile, the inherent high robustness property of the CSMC is fully preserved in the proposed controller. Where the learning algorithm continuously adjusts the closed-loop response based on the most recent history of closed-loop stability. Thus, the stability and convergence analysis of the RSMLC is proved rigorously. Simulation studies have been conducted for a piezoelectric actuator (PEA) system, as a prototype of MSA. For comparison reasons, these results are presented correspondingly with CSMC results to demonstrate the effectiveness of the proposed controller over the CSMC.
\end{abstract}

Keywords: Robust Control, Sliding Mode Control, Learning Control, Micro-Scale Actuator, Piezoelectric Actuator.

\section{Introduction}

Micro/nano-positioning devices actuated by piezoelectric actuators have been extensively employed in fields such as optical scanning, biomedicine, precision manufacturing, aerospace where ultrahigh precision is required [1]. For example, microinjection systems [2], atomic precision imaging [3], micromanipulators and microgrippers [4] and many others [5].

Piezo-driven actuators have a number of benefits that can be achieved by the applications, for instance high precision motion (i.e., nanometers or below), fast transient response, high blocking force range of $\mathrm{N}$ to $\mu \mathrm{N}$ and excellent electro-mechanical coupling and properties (i.e., lightweight, high stiffness, and low energy consumption) [6]. However, the nonlinear nature of PEAs (i.e., typically suffering from creep, and hysteresis) is an obstacle that led to deter their usage. Particularly, the hysteresis effect caused by the input and output nonlinear relationship in the PEA can lead to severe position error up to $15 \%$ of the travel range of the piezodrive platform [7]. Overcoming this nonlinearity is essential to attain the merits of PEA and make it works effectively. A variety of control strategies have been employed to cope with this issue and achieved the required precision in piezo-driven positioning systems. A survey of the most recent control techniques utilized for PEAs and their applications is reported by [4, [6], [9]. Controllers can be designed as feedforward, feedback, and a combination of both [4]. As a model-based control method, in feedforward control, the hysteresis model is essential [6]. Deriving an accurate hysteresis model is not only a complex process but also is a timeconsuming process [10].

Alternatively, feedback control is being adopted in numerous applications for piezo-driven micro/nano-positioning systems, in particular, sliding mode control strategies [11]. By considering hysteresis to be a bounded disturbance, SMC is employed to attenuate the hysteresis effect and achieve the desired performance [12-14]. However, the inherited chattering phenomenon in SMC is significantly deterring its applications and could excite undesirable dynamics behavior of the systems [15]. To mitigate the chattering effect, numerus control techniques have been proposed in the literature. Among these are the high order SMC, terminal SMC and adaptive SSMC [13], [16], [17]. Recently, a new SMC approach namely robust sliding mode based learning control (RSMLC) has been proposed in [18]. The RSMLC not only mitigate the chattering issue but also no knowledge of system uncertainties and external disturbance is required in 
the control design. As a result, neither the state observer nor the hysteresis model are necessary in this control structure. Such benefits not only simplify the control design procedure, but also facilitate its practical applications. Besides, the system stability in the RSMLC is achieved by learning technique, which is simply done by adjusting the closed-loop response continuously based on its previous sample. In this paper, we have exploited these advantages to control a typical MSA to achieve a high precision tracking control, namely piezo-driven positioning systems. Finally, the control approach was validated by performing a simulation study on a piezo-driven platform as a prototype of MSA systems.

The rest of the paper is organized as follows. The dynamics model is elaborated in Section 2. The formulation of RSMLC for PEA is presented in Section 3. Furthermore, Section 4 deals with system stability and convergence analysis. The simulation studies are conducted in Section 5. Finally, conclusions are presented in Section 6 .

\section{Modelling of MSA}

As a prototype of MSA, a piezoelectric actuator has been employed in this work. To this end, the selected piezo-driven micro stage is depicted in Figure 1. It is driven by a built-in PZT micro-actuator (i.e., type of P-725, Polytec PI) which is the core of the PEA system. The flexure guide will then drive the moving mechanism of the micro stage to follow the desired trajectories. It has the ability to travel as long as 12.5 micrometers with a high moving resolution that can reach a few nano-scales.

Therefore, a high precision positioning sensor (i.e., capacitor sensor) is attached to measure and transmit the instantaneous position of the moving stage. It is well known that the main obstacle in these actuators is the inherent hysteresis. In order to overcome this issue, the effect of hysteresis nonlinearity was considered as a disturbance over a linear system [19].

According to previous work [12], the mathematical model of PEA is described by the following transfer function

$$
F(s)=\frac{x(s)}{u(s)}=\frac{k \omega_{n}^{2}}{s^{2}+2 \xi \omega_{n} s+\omega_{n}^{2}}
$$

where $k^{\prime} \xi \omega_{n}$ are the stiffness, damping ratio and natural frequency, respectively. Meanwhile, $s$ stands for Laplace operator, ${ }_{u}$ is the control input and ${ }_{x}$ is the output displacement to be controlled. To facilitate the control design, Eq. 1 has been rewritten in the following form

$$
\ddot{x}+c_{1} \dot{x}+c_{2} x+\Psi=c_{3} u
$$

where $c_{1}=2 \xi \omega_{n}^{\prime} \quad c_{2}=\omega_{n}^{2}, \quad c_{3}=k \omega_{n}^{2}$ and the variable $_{\psi}$ indicates the lumped system perturbation which includes modelling errors, system uncertainties and external disturbance.

It should be noted that these perturbations are unknown neither their values nor their boundaries. Thus, it is one merit of employing the RSMLC for highly nonlinear systems such as PEA. For further analysis, the state space format of Eq. 2 is expressed as

$$
\dot{x}_{2}=-c_{1} x_{2}-c_{2} x_{1}-\Psi+c_{3} u
$$

where $x_{1}=x$ and $x_{2}=\dot{x}$. In order to proceed with the control design, the nominal parameters of the PEA system have been identified. To this end, practical identification was carried out in previous work [11], [20]. Hence, the parameter values are reported as $1810,1 * 10^{7}$ and $3 * 10^{6}$ for $c_{1}, c_{2}$ and $c_{3}$, respectively. For clarification purposes, we should emphasise that the hysteresis model is not considered in this paper; however, it is embedded with the unknown system perturbation $\psi$ which will be dealt with blindly by the proposed controller.
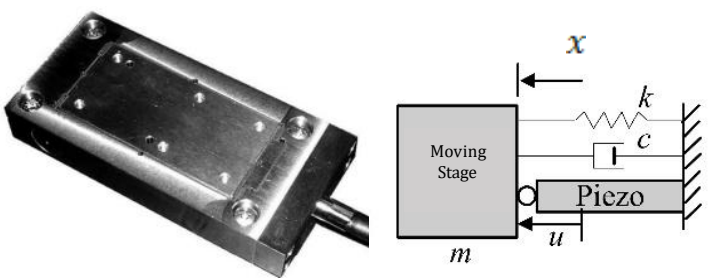

Figure 1: Micro positioning actuator with internal modelling structure.

\section{RSMLC Formulation for PEA}

The objective of the proposed controller is to achieve high precision PEA trajectory tracking in the circumstance of the external disturbance, unmodelled inherent nonlinear hysteresis and system parameters variation. To this end, the RSMLC design will elaborate as follows.

First, the tracking error is defined as:

$$
e(t)=x(t)-x_{r}(t)
$$

where $x(t)$ is the measurable displacement of the micro stage platform, $x_{r}(t)$ is the tracking reference and $t$ is the time parameter, which will be omitted for the sake of simplicity. Next, the sliding surface is selected as follows:

$$
\sigma=\dot{e}+\lambda e
$$


where $\lambda \in \mathcal{R}$ and $\lambda>0$. Then, the time derivative of

(5) is expressed by the formula

$$
\dot{\sigma}=\phi+c_{3} u
$$

where $\phi=-c_{1} x_{2}-c_{2} x_{1}-\Psi-\ddot{x}_{r}+\lambda e$.

The adopted robust sliding-based learning controller in this paper is given by [21]:

$$
u(t)=u(t-\tau)-\delta u(t)
$$

where $\delta u(t)$ is the iterative learning part defined as

$$
\delta u(t)=\left\{\begin{array}{rr}
\frac{1}{c_{3} \sigma}\left(\eta_{1} \hat{\dot{v}}(t-\tau)+\eta_{2}|\hat{v}(t-\tau)|\right), \sigma & \neq 0 \\
0 & \sigma=0
\end{array}\right.
$$

where $\tau$ is the time delay, $\eta_{1}$ and $\eta_{2}$ are the control parameters to be designed and $\dot{v}(t)$ is the candidate Lyapunov function defined as

$$
v(t)=\frac{1}{2} \sigma^{2}
$$

meanwhile, $\quad \hat{\dot{v}}(t-\tau)=(v(t)-v(t-\tau)) / \tau$ is the delayed and estimated value of $\dot{v}(t)$.

Remark 1: An insightful view of the proposed controller in (7) and (8), it can be deduced that when $\sigma(t) \neq 0$ the control signal will be continuous due to the learning part. Meanwhile, when $\sigma(t)=0$ the only previous signal $u(t-\tau)$ would be in charge. This process led to a continuous control effort, and hence the inherent chattering drawback in the CSMC method will be significantly alleviated. This is one merit of using RSMLC (i.e., chattering free control), which results in protecting and extending the lifespan of the PEAs.

Remark 2: The time delay $\tau$ is generally chosen to be as small as the implementation hardware is allowed. For instance, it could be selected as one sampling period.

\section{Convergence Analysis}

In order to prove the whole system stability, the following Lemma has been introduced:

Lemma: Consider the PEA model in (3) under the proposed RLSMC law in (7) with correction term in (8), then a zero asymptotic convergence of the tracking error $e_{e}$ in (4) can be guaranteed.

\section{Proof:}

Recalling the candidate Lyapunov function $v(t)$ from Eq. 9, then the time derivative of $v(t)$ along the system trajectory can be achieved as:

$$
\begin{aligned}
\dot{v}(t) & =\sigma(t) \dot{\sigma}(t) \\
& =\sigma\left[\phi+c_{3} u(t)\right] \\
& =\sigma\left[\phi+c_{3} u(t-\tau)\right]-\sigma c_{3} \delta u(t)
\end{aligned}
$$

substituting the learning term $\delta u(t)$ from Eq. 8 into Eq. 10 yields

$$
\begin{aligned}
\dot{v}(t)= & \sigma\left[\phi+c_{3} u(t-\tau)\right]- \\
& \sigma c_{3}\left\{\frac{1}{c_{3} \sigma}\left(\eta_{1} \hat{v}(t-\tau)+\eta_{2}|\hat{v}(t-\tau)|\right)\right\}
\end{aligned}
$$

Since $\dot{v}(t)=\sigma\left[\phi+c_{3} u(t)\right]$, then it can be deduced that

$$
\dot{v}(t, t-\tau)=\sigma\left[\phi+c_{3} u(t-\tau)\right]
$$

Substitute (12) into (11) yields

$$
\begin{aligned}
\dot{v}(t)= & \dot{v}(t, t-\tau)- \\
& \left(\eta_{1} \hat{v}(t-\tau)+\eta_{2}|\hat{v}(t-\tau)|\right)
\end{aligned}
$$

the following assumption is introduced to proceed in system proof.

\section{Assumption:}

The research assumption is that $\dot{v}(t)$ is a continuous function during the interval ${ }_{\tau}$. It should be noted that this assumption is valid since ${ }_{\tau}$ would be a very short period.

According to the Lipschitz-like condition in [18], the following inequality is held

$$
|\dot{v}(t, t-\tau)-\dot{v}(t-\tau)|<\frac{1}{M}|\hat{\dot{v}}(t-\tau)|
$$

where, $M$ is a constant number chosen to satisfy specific conditions as will be illustrated later. By adding $(\dot{v}(t-\tau)-\dot{v}(t-\tau))$ to (13) and considering (14) into account, we obtain

$$
\begin{aligned}
\dot{v}(t)< & \frac{1}{M}|\hat{\dot{v}}(t-\tau)|+\dot{v}(t-\tau) \\
& -\left(\eta_{1} \hat{\dot{v}}(t-\tau)+\eta_{2}|\hat{\dot{v}}(t-\tau)|\right) .
\end{aligned}
$$

Indeed, there are two possible cases in (15) that should be investigated.

The first case when $\dot{v}(t-\tau)>0$ :

$$
\begin{aligned}
& \dot{v}(t)<\dot{v}(t-\tau)+\left(\frac{1}{M}-\eta_{1}\right)|\hat{\dot{v}}(t-\tau)| \\
& -\eta_{2}|\hat{\dot{v}}(t-\tau)|
\end{aligned}
$$

If the control parameters are selected to satisfy the following conditions 


$$
M, \beta, \alpha \in \mathcal{R}, M \gg 1_{\eta_{1}}>\frac{1}{M}
$$

then Eq. 15 is simplified into

$$
\dot{v}(t)<\dot{v}(t-\tau) \text {. }
$$

which implies that when $\dot{v}(t-\tau)>0$ it keeps decreasing due to the learning controller. In other words, the proposed controller constantly reduces the instantaneous derivative of the Lyapunov function from positive to negative value. The next possible case is that when $0<\dot{v}(t-\tau)$, in this case, we can directly conclude that:

$$
\dot{v}(t)<0
$$

hence, the system stability proof is completed.

Remark 3: For comparison reason, the CSMC is designed based on boundary layer technique as follows:

$$
u_{s m c}=u_{n}+k_{r} \operatorname{sat}\left(\frac{s_{c s m c}}{\Delta}\right)
$$

where $k_{r}>0, \Delta$ is the boundary layer thickness and sat is the saturation function which is used to replace the signum function to alleviate the chattering effect [26]. Meanwhile, the sliding variable is given as $s_{\text {csmc }}=\dot{e}+\lambda e$, where $e$ is the tracking error defined in Eq. 4 . Once the sliding condition is satisfied $s_{c s m c}=0$ we can easily deduce $u_{n}$ as

$$
u_{n}=\frac{1}{c_{3}} \ddot{x}+\frac{c_{2}}{c_{3}} c_{1} \dot{x}+\frac{c_{2}}{c_{3}} x
$$

\section{Results and Discussion}

In this section, the simulation studies are carried out to validate the RSMLC performance on the PEA system. The tracking results for the nominal PEA model is presented first, and then the robustness of RSMLC against plant uncertainties is investigated by showing the system performance under these effects. To show the superiority of the proposed controller, we compare the tracking performance of the RSMLC with a CSMC in Eq. 19 for both tests.

\subsection{Tracking Performance}

The reference signal $x_{r}(t)$ is set as a swept sinusoidal reference with an amplitude of $1 \mu \mathrm{m}$ and variable frequency with time from $1 \mathrm{~Hz}$ to $10 \mathrm{~Hz}$. It should be noted that such trajectories would excite the internal hysteresis nonlinearities. Thus, the tracking error would increase proportionally.

Figure 2.a shows the tracking response under the designed RSMLC and SMC under nominal conditions. Generally, we can see from the upper plot that the tracking performance for both methods is acceptable.

The zoomed area, however, showed the chattering issue is significant under SMC. Meanwhile, the chattering effect is remarkably mitigated under the RSMLC. To emphasize this fact Figure 2.b is presented, which indicates the control efforts of each controller. Due to the switching part in the SMC design, the chattering is clear and significant which can lead to minimizing the life span of these precious actuators and deteriorate their performance particularly in the presence of system perturbation, which will demonstrate in the next subsection. On the other hand, we can see from Figure 2.b that the proposed controller is effectively handled this issue.

This means it provides an alternative control method that preserves the SMC robustness and overcome its limitations.
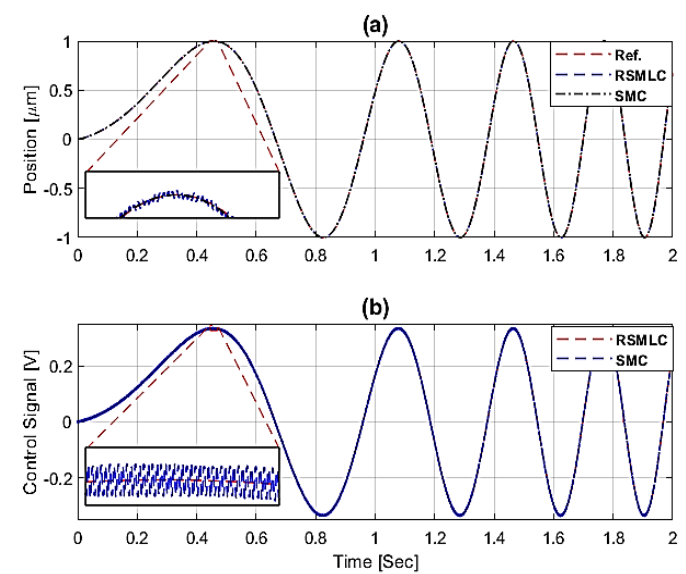

Figure 2 Tracking performance of RSMLC and SMC for nominal PEA model

\subsection{Robustness Verification}

In order to verify that the proposed controller inherits the robustness of the sliding mode control method, we multiply the system parameters by a factor of 1.5 to represent a perturbed PEA model.

In practical terms, the perturbation of the system can be due to many factors, such as the payload, the change of atmosphere of the system and working circumstances. Thus, we run the simulation for both controllers and the tracking response is plotted in Figure 3. We can see that both controllers can handle this variation in system parameters, which is the main advantage of the SMC methodology. However, with further investigation, we can see that RSMLC has much less oscillations than CSMC due to the variation in mass. 
In theory, these results are claimed by the control design. Unlike CSMC, the internal structure of RSMLC does not contain an explicit switching part, leading to the results shown in Figure 3. b and hence it can be classified as chattering free control method. Consequently, the overall position profile of the PEA as shown in the upper plot of Figure 3 did not have a significant difference compared to that of Figure 2 (i.e., tracking performance under nominal conditions). This not only verifies the strong robustness of the RSMLC with respect to the system parameters variations, but also the smoothness of the control signal.
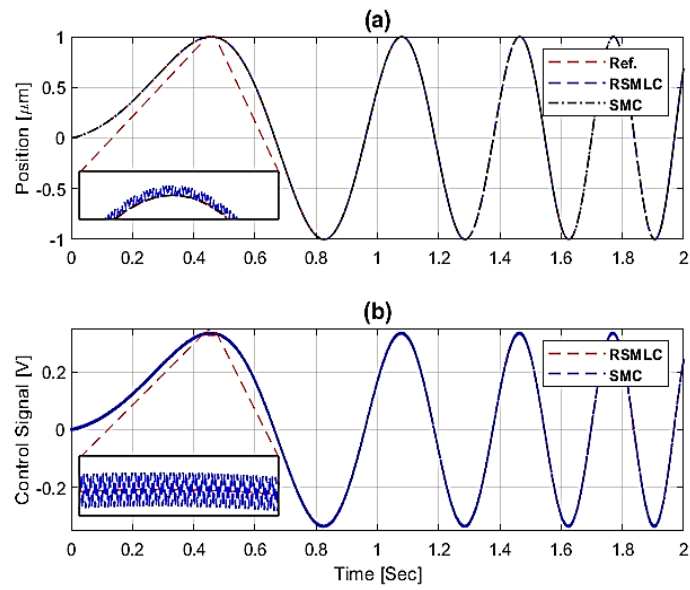

Figure 3 Tracking performance of both controllers in the presence of system uncertainties.

\section{Conclusions}

In this paper, the design of RSMLC for a class of MSA systems was presented. Firstly, the PEA mathematical model was derived into a secondorder differential equation and then it was written in the form of state-space to facilitate the control design. Next, the RSMLC method was formulated the MSA system formulated. The system stability, on the other hand, was rigorously proofed via the Lyapunov criteria. Finally, the proposed controller was applied for the plant models of the PEA system. The simulation results were presented to validate the merits of the RSMLC controller in the absence and presence of system perturbations. Furthermore, it was compared with the CSMC controller. These results were strongly validated the theory claims in the terms of tracking performance, system robustness and chattering mitigation.

\section{References}

[1] Y. Liu, J. Deng, and Q. Su, "Review on multidegree- of-freedom piezoelectric motion stage," IEEE Access, vol. 6, pp. 59986-60004, 2018.

[2] Y. Wei and Q. Xu, "Design and testing of a new force-sensing cell microinjector based on small- stiffness compliant mechanism," IEEE/ASME Trans. Mechatronics, vol. 26, no. 2, pp. 818-829, 2020.

[3] A. Alipour, S. O. R. Moheimani, J. H. G. Owen, E. Fuchs, and J. N. Randall, "Atomic precision imaging with an on-chip scanning tunneling microscope integrated into a commercial ultrahigh vacuum STM system," J. Vac. Sci. Technol. B, Nanotechnol. Microelectron. Mater. Process. Meas. Phenom., vol. 39, no. 4, p. 40603, 2021.

[4] Z. Lyu and Q. Xu, "Recent design and development of piezoelectric-actuated compliant microgrippers: A review," Sensors Actuators A Phys., vol. 331, p. 113002, Nov. 2021, doi: 10.1016/J.SNA.2021.113002.

[5] M. Maroufi, A. G. Fowler, and S. O. R. Moheimani, "MEMS for nanopositioning: Design and applications," J. Microelectromechanical Syst., vol. 26, no. 3, pp. 469-500, 2017.

[6] D. V Sabarianand, P. Karthikeyan, and T. Muthuramalingam, "A review on control strategies for compensation of hysteresis and creep on piezoelectric actuators based micro systems," Mech. Syst. Signal Process., vol. 140, p. 106634, 2020.

[7] Z. Li, J. Shan, and U. Gabbert, "Inverse compensation of hysteresis using KrasnoselskiiPokrovskii model," IEEE/ASME Trans. Mechatronics, vol. 23, no. 2, pp. 966-971, 2018.

[8] W. Wei, P. Xia, W. Xue, and M. Zuo, "On the disturbance rejection of a piezoelectric driven nanopositioning system," IEEE Access, vol. 8, pp. 74771-74781, 2020.

[9] N. N. Son, C. Van Kien, and H. P. H. Anh, "A review on control strategies for compensation of hysteresis and creep on piezoelectric actuators based micro systems," Eng. Appl. Artif. Intell., vol. 87, p. 103317, 2020.

[10] H. Habibullah, "30 Years of Atomic Force Microscopy: Creep, Hysteresis, Cross-coupling, and Vibration Problems of Piezoelectric Tube Scanners," Measurement, p. 107776, 2020.

[11] A. Al-Ghanimi, J. Zheng, and Z. Man, "Robust and fast non-singular terminal sliding mode control for piezoelectric actuators," IET Control Theory Appl., vol. 9, no. 18, 2015, doi: 10.1049/ietcta.2015.0401.

[12] A. Al-Ghanimi, J. Zheng, and Z. Man, "A fast nonsingular terminal sliding mode control based on perturbation estimation for piezoelectric actuators systems," Int. J. Control, vol. 90, no. 3, 2017, doi: 10.1080/00207179.2016.1185157.

[13] G. Wang and Q. Xu, "Adaptive Terminal Sliding Mode Control for Motion Tracking of a Micropositioning System," Asian J. Control, 2018.

[14] J. P. Mishra, Q. Xu, X. Yu, and M. Jalili, "Precision position tracking for piezoelectric-driven motion system using continuous third-order sliding 
mode control," IEEE/ASME Trans. Mechatronics, vol. 23, no. 4, pp. 1521-1531, 2018.

[15] Z. Zheng, H. Zhang, and D. T. Vo, "ANALYSIS OF MODEL-FREE SLIDING MODE CONTROL OF PERMANENT MAGNET SYNCHRONOUS MOTOR," Int. J. Mechatronics Appl. Mech., no. 8, pp. 217224, 2020.

[16] A. Al-Ghanimi, J. Zheng, A. Aldhalemi, J. Khawwaf, and Z. Man, "Second-order terminal sliding mode control based on perturbation estimation for nanopositioning stage," IET Cybersystems Robot., vol. 2, no. 4, pp. 161-167, 2020.

[17] L. Qiao and W. Zhang, "Adaptive Second-Order Fast Nonsingular Terminal Sliding Mode Tracking Control for Fully Actuated Autonomous Underwater Vehicles," IEEE Ocean. Eng., vol. 44, no. 2, pp. 363-385, Apr. 2019, doi: 10.1109/joe.2018.2809018.
[18] Z. Man, S. Khoo, X. Yu, and J. Jin, "A new sliding mode-based learning control scheme," in Industrial Electronics and Applications (ICIEA), 2011 6th IEEE Conference on, 2011, pp. 19061911.

[19] E. Sariyildiz, R. Oboe, and K. Ohnishi, "Disturbance observer-based robust control and its applications: 35th anniversary overview," IEEE Trans. Ind. Electron., vol. 67, no. 3, pp. 20422053, 2019.

[20] J. Zheng and M. Fu, "Saturation control of a piezoelectric actuator for fast settling-time performance," IEEE Trans. Control Syst. Technol., vol. 21 , no. 1 , pp. 220-228.

[21] Z. Man, C. Zhang, J. Jin, and others, "A new sliding mode-based learning control for uncertain discrete-time systems," in Control Automation Robotics |\& Vision (ICARCV), 2012 12th International Conference on, 2012, pp. 741-746. 